\title{
El proceso de diseño de un estudio narrativo sobre noviolencia centrado en la escucha. Desafios éticos, socio-históricos y metodológicos del trabajo con relatos de lucha social en Chile*
}

Designing a narrative study of nonviolence centered on listening: The ethical, technical, and methodological challenges of working with life stories of social struggle in Chile

\author{
Carolina Muñoz-Proto \\ Alejandro Ancapichún-HernándeZ \\ René SQuella-Soto \\ Pontificia Universidad Católica de Valparaíso (Chile) \\ cpmproto@gmail.com (CHILE)
}

Recibido: 14.062018

Aceptado: 12.12.2019

\section{RESUMEN}

El giro de las ciencias sociales hacia lo narrativo ha permitido abordar la experiencia humana en su dimensión socio-histórica y psicosocial a través del estudio de relatos biográficos, relatos breves de experiencia, y otras formas de narración (Bernasconi, 2011; Capella, 2013; Chase, 2015; Cornejo, Mendoza y Rojas, 2008; Daiute y Lightfoot, 2004; Doucet y Mauthner, 2008; Josselson y Lieblich, 1993). A la luz de estos avances, presentamos aquí el proceso de diseño teórico-metodológico de un estudio sobre no-violencia y activismo ciudadano que se centró en las historias de vida de activistas de la tercera edad quienes participaron de la izquierda armada chilena en su juventud. A través de la discusión del proceso de diseño del estudio en cuestión, el artículo aborda los desafíos éticos, relacionales y técnicos que enfrentamos cuando optamos por una práctica investigativa centrada en la recopilación y análisis de relatos de lucha social en un contexto de posconflicto.

El diseño resultante combina técnicas reconocidas dentro del enfoque biográfico con una adaptación original de la Guía de Escucha, una técnica feminista desarrollada en la década de 1980 (Gilligan, Spencer, Weinberg y Bertsch,

*Financiado por CONICYT FONDECYT 11150808

EMPIRIA. Revista de Metodología de Ciencias Sociales. N. ${ }^{\circ} 45$ enero-abril, 2020, pp. 143-163.

ISSN: 1139-5737, DOI/ empiria. 43.2020.26307 
2003), aún sin gran desarrollo en contextos hispanos. Discutimos dos aspectos de la Guía de Escucha y su aplicación al estudio: la concepción dinámica y relacional de la subjetividad narrada y la práctica de una forma de escucha activa, recursiva y reflexiva orientada por preguntas. Tras describir los antecedentes y objetivos del estudio en cuestión, discutimos en profundidad los desafíos del proyecto en sus dimensiones éticas, relacionales y técnicas. Se presentan luego los marcos teórico-metodológicos con los que se ha enfrentado dichos desafíos, dando paso a la descripción detallada del diseño resultante y sus diversos momentos del proceso de producción y análisis de relatos. Discutimos también las condiciones ideales para la implementación de dicho diseño. En su conjunto, el artículo contribuye al desarrollo de la investigación narrativa y al diálogo sobre los desafíos metodológicos que caracterizan los estudios narrativos realizados en contextos de posconflicto y basados en la práctica de la escucha.

\section{PALABRAS CLAVE}

Estudios Narrativos; Relatos de Lucha social; No-violencia; Guía de Escucha; Escucha.

\section{ABSTRACT}

The narrative turn in the social sciences has allowed for the exploration of human experience in its socio-historical and psychosocial dimensions through the study of life stories, brief accounts of experience, and other forms of narration (Bernasconi, 2011; Capella, 2013; Chase, 2015; Cornejo, Mendoza y Rojas, 2008; Daiute y Lightfoot, 2004; Doucet y Mauthner, 2008; Josselson y Lieblich, 1993). In light of these developments, we present the process of designing a study of nonviolence and grassroots activism which was centered on the life stories of senior activists who, in their youth, took part in the armed movements of the chilean left. Through a discussion of the process of design of said study the article addresses the ethical, relational and technical challenges scholars face when choosing a research practice based on the gathering and analysis of stories of social struggle in post-conflict settings.

The resulting design combines well-established techniques from biographical studies with an original adaptation of the Listening Guide, a feminist technique developed in the decade of the 1980s (Gilligan, Spencer, Weinberg y Bertsch, 2003), yet without much development in Hispanic contexts. We discuss two aspects of the Listening Guide and their application to the study: A dynamic and relational understanding of narrated subjectivities and the practice of a form of active, recursive and reflective listening that is guided by questions. After describing the background and objectives of the study, we discuss its ethical, relational and technical challenges in detail. We then present the theoretical and methodological frameworks with which we have addressed said challenges, 
setting the stage for a detailed description of the various moments of production and analysis of life stories that make up the resulting design. We discuss, as well, the ideal conditions for the implementation of said design. In this way, the article contributes to the development of narrative research and to current dialogue regarding the methodological challenges that arise in narrative studies that are carried out in post-conflict settings and that are centered on the practice of listening.

\section{KEY WORDS}

Narrative Studies; stories of social struggle, Listening Guide; Nonviolence; Listening.

\section{INTRODUCCIÓN}

El giro de las ciencias sociales hacia lo narrativo ha abierto la posibilidad de abordar la experiencia humana en su dimensión socio-histórica y psicosocial a través del estudio de relatos biográficos, relatos breves de experiencia, y otras formas de narración (Atkinson, 2001; Bernasconi, 2011; Bolívar y Domingo, 2006; Capella, 2013; Chase, 2015; Cornejo, 2006; Cornejo, Mendoza y Rojas, 2008; Daiute y Lightfoot, 2004; Doucet y Mauthner, 2008; Josselson y Lieblich, 1993). En los estudios psicosociales de la paz y los conflictos, vemos en los últimos quince años prometedoras aplicaciones de estos abordajes al estudio de las crisis socio-políticas (Andrews, 2007; 2014); el desarrollo de las personas en contextos de violencia (Daiute, 2010; 2013); las dimensiones psicológicas de la paz (De la Rey, 2011; Law y Bretherton, 2015); y el rol de la psicología de la memoria, la justicia y la reconciliación en posconflicto (Duncan, Stevens y Sonn, 2012), entre otras.

A la luz de estos avances, presentamos aquí el proceso de diseño metodológico de un estudio centrado en las historias de vida de activistas de la tercera edad $_{2}$ quienes participaron de la izquierda armada chilena en su juventud. El artículo responde a las siguientes preguntas: ¿Qué desafíos éticos y técnicos conllevan la recopilación y análisis de relatos de lucha social en un contexto de posconflicto? ¿Qué pasa cuando nos disponemos a escuchar relatos de vida de personas diferentes a nosotros? ¿Desde dónde escuchamos y con qué técnicas? ¿Qué marcos teórico-metodológicos y qué diseños pueden dar respuesta a estas interrogantes?

Tanto el estudio mismo como los objetivos del artículo se fundamentan en el rol que los relatos biográficos y otras narrativas de experiencia desempeñan en tanto formas de construir y comunicar el conocimiento humano (Bruner, 1990). Si bien el giro narrativo en psicología ha configurado al relato como objeto de estudio, en general se mantiene una cierta reverencia disciplinar hacia lo que Bruner (1990) denominara pensamiento lógico-formal, aún visto como la forma 
más válida de conducir, integrar y comunicar experiencias investigativas. Dado que la gran mayoría de los requerimientos institucionales y los códigos de ética en investigación académica están pensados desde una lógica cuantitativa (Flick, 2014; Lincoln, 2012), buscamos aportar al desarrollo de las dimensiones éticometodológicas de la investigación cualitativa sobre fenómenos relacionados a la justicia social (Anderson y Muñoz-Proto, 2016; Fine, 2012).

El propósito del artículo es, entonces, contribuir al desarrollo de la investigación narrativa, describiendo en profundidad los desafíos éticos y técnicos que caracterizan a un estudio sobre lucha social. Comenzamos por describir los antecedentes y objetivos del estudio, para luego identificar los desafíos del proyecto en sus dimensiones éticas y técnicas. Tras presentar los marcos teóricometodológicos con lo que proponemos enfrentar dichos desafíos, concluimos con una discusión que aporta una mayor comprensión sobre cómo el diseño al que arribamos responde a los desafíos que se nos fueron presentando.

\section{EL ESTUDIO}

El estudio en cuestión pertenece a una línea de investigación sobre el activismo ciudadano no-violento y su rol en la construcción de una paz sostenible (Kool, 1993; Muñoz-Proto y Opotow, 2012; Muñoz-Proto, 2014; Muñoz Proto, Devoto Lyon, Villar Castillo, C. y Battistella, 2013; Reardon y Cabezudo, 2012). Desde este marco, al abordar las experiencias de activistas por la justicia social, nos focalizamos en sus procesos de aprendizaje, desarrollo y cambio: ¿Qué experiencias orientan a las personas a enfrentar de distintos modos las injusticias y opresiones que enfrentan a lo largo de sus vidas?; ¿Qué experiencias formativas sensibilizan a las personas hacia una u otra metodología de acción política?; ¿Qué búsquedas identitarias de juventud encuentran respuesta en uno u otro modelo de lucha social?

Tales preguntas están insertas en el contexto socio-histórico de Chile: una sociedad en posconflicto que vivió el proceso de movilización popular del gobierno de Salvador Allende y una dictadura cívico militar entre 1973 y 1989 (Donoso Jiménez, 2013; Garcés, 2011; Guerrero Antequera, 2006). El país actualmente muestra señales de gran malestar y altos niveles de conflictividad ambiental, política y social (Jaimovich, González, Calbucura, del Valle, y Maldonado, 2018), además de demandas por reformas a sistemas públicos y privados (Bellei, Cabalin y Orellana, 2014; Garcés, 2012; Hatibovic Díaz, Sandoval Moya y Cárdenas Castro, 2012; Martínez, Peñaloza y Valenzuela, 2012). 


\section{DESAFÍOS TÉCNICOS, RELACIONALES Y ÉTICOS DEL ESTUDIO}

Fue el año 2015 que comenzamos el proceso de sondeo y diseño del estudio. A continuación describimos los principales desafíos técnicos, relacionales y éticos que enfrentamos, centrándonos en el proceso de definición de criterios muestrales, así como nuestro propio posicionamiento frente a los relatos y los alcances éticos del estudio.

\subsection{Criterios de inclusión o ¿A quiénes escuchar?}

Los hallazgos de estudios anteriores sobre activismo por la paz en los países de los Andes (Muñoz-Proto, 2014; Anderson y Muñoz-Proto, 2016) nos orientaban hacia el estudio de personas con experiencia directa con la lucha armada y la acción no-violenta en contextos estudiantiles, territoriales y sindicales. Para traducir esta idea difusa en un diseño concreto, realizamos 10 entrevistas con informantes claves y porteros de comunidades (Sixsmith, Boneham, y Goldring, 2003) relacionadas con el activismo ciudadano de los sectores urbanos de izquierda de la zona central del país. Dialogamos así con líderes religiosos, profesionales de la salud mental y líderes comunitarios sobre los objetivos tentativos de nuestro estudio y las implicancias de trabajar con relatos en el Chile actual. Estas personas nos informaron sobre la necesidad de documentar el tránsito el país desde el activismo ciudadano del Chile del siglo XX hacia nuevas formas aún en construcción, con especial interés en los efectos de la dictadura en dicho proceso (Goicovic, 2004; Lira, 2010, 2013; Moulián, 2002; Salazar, 2013, 2015).

Con este tránsito en mente, decidimos focalizar el estudio en las experiencias de activistas de la tercera edad que en su juventud participaron de la izquierda armada y que a lo largo de las décadas continuaron participando activamente de una serie de movimientos centrales a la historia reciente de Chile (Lozoya, 2012; Pérez, 2012). Las características específicas de la muestra se esclarecieron durante el proceso de sondeo y las primeras entrevistas de sondeo, en particular el criterio etario. Al comparar las trayectorias políticas de personas entre 50 y 80 años, decidimos excluir de la muestra a quienes se iniciaron en su vida política en reacción a la dictadura. Esto, con el fin de focalizarnos en las trayectorias de quienes se iniciaron en la vida política antes del golpe militar, en un contexto propositivo de alzamiento de diversos movimientos de izquierda. Fue también durante el proceso que se revelaron diferencias significativas en las trayectorias de hombres y mujeres de dicha generación, así como la naturaleza principalmente masculina de la población. Finalmente, el proceso de sondeo mostró que una gran mayoría de las trayectorias de la población del estudio incluían una actual participación causas y movimientos no-armados en el área socio-ambiental, las comunicaciones y lo educacional.

Como resultado, focalizamos el estudio en las experiencias de activistas entre 60 y 80 años de la zona central del país con cuatro características comunes: 
(1) participación sostenida en movimientos, partidos, causas y/o organizaciones sociales y políticas desde su juventud hasta el día de hoy durante (a) el gobierno de la coalición de partidos de izquierda liderada por Salvador Allende, (b) la dictadura, (c) la transición a la democracia y (d) la post-dictadura.; (2) iniciación de sus vidas políticas antes del inicio de la dictadura en 1973; (3) participación actual en causas y movimientos no-armados en el área socio-ambiental, comunicaciones y/o educación en roles activos; y (4) participación en una organización político-militar de izquierda en roles de combate, logística y/o apoyo directo por más de tres meses entre durante los años en que dichas organizaciones fueron actores claves del escenario político chileno (1970 y 1995).

\subsection{Posicionamiento ante los relatos}

El proceso de sondeo y las primeras entrevistas evidenciaron que la temática y formato narrativo del estudio generaban diversos desafíos de posicionamiento ante los relatos, tanto para los participantes del estudio como para los miembros del equipo de investigación. Así mismo, se nos evidenció el desafío de significar la violencia estructural, la lucha armada, la violencia política y la no- violencia activa, tanto al narrar como al escuchar los relatos de lucha social.

Como equipo de investigación y audiencia directa de los relatos, nos encontrábamos con capítulos y escenas de la historia chilena contadas desde las minucias de estas vidas. Nos encontrábamos con matices, hechos y perspectivas a veces conocidas, a veces muy diferentes a lo que ya conocíamos de la versión oficial de la historia del país; versión que había llegado a nosotros por los medios de comunicación, las historias familiares y los productos del proceso de verdad y reconciliación. Como han comentado otros autores (Reyes, Muñoz y Vázquez, 2013) este aprendizaje evocaba en nosotros reacciones afectivas en las que topábamos a veces con lo nuevo y a veces con reacciones que se han instalado como aceptables (Reyes et al., 2013), como la despolitización del diálogo. Ante esto, a veces aparecía la dificultad de escuchar y comprender experiencias de momentos históricos que no hemos compartido. Otras veces surgía la dificultad de escuchar experiencias que no necesariamente validamos o con las que nos es difícil empatizar. Todo el equipo compartía la incomodidad de analizar y disectar relatos de otras personas. Experimentábamos también el esfuerzo de escuchar y comprender en profundidad, a pesar de los prejuicios y las creencias propias y más allá de lugares comunes y de las autocensuras de quienes nos estaban contando sus vidas.

El ejercicio de relatar generaba desafíos para nuestros participantes, situados en la sociedad chilena como figuras controversiales cuestionadas, por la derecha, el Estado dictatorial, el Estado transicional, los sectores de centroizquierda y los propios compañeros de lucha. Se veían en la dificultad de elegir desde dónde y para quién narrar. Fuimos notando cómo el entusiasmo y pasión por compartir el propio relato convivía con una dificultad de articular experiencias personales más allá de ciertos lugares comunes, de ciertas líneas ideológicas o de ciertos 
tabúes sobre la violencia y la acción. Ya desde las primeras entrevistas notamos una mayor elaboración de los relatos en torno a las vivencias en dictadura, en especial las vivencias de victimización por parte del Estado, en desmedro de otros momentos de la vida. Todas estas dinámicas percibidas -tal vez imaginadas o malinterpretadas por nosotros- hacían más compleja nuestra experiencia como audiencia.

Otro desafío fue ir digiriendo las reacciones de la audiencia de nuestro estudio (colegas, porteros de comunidades claves, familiares, amigos, colaboradores, pares y empleadores) entre quienes reconocimos algo así como un "dime qué investigas y te diré quién eres." El mero hecho de estar llevando a cabo el estudio funcionaba como una heurística para posicionarnos en ciertos roles o espacios políticos predefinidos en el imaginario chileno: la defensa de la lucha armada, la defensa de una cierta concepción de los derechos humanos, la militancia de ultraizquierda, la traición al sentido común de centro-derecha, entre otros. Este posicionamiento no elegido contrastaba con las experiencias que algunos miembros del equipo de investigación habían tenido en otros países. En Colombia o en Estados Unidos, por ejemplo, la investigación con comunidades de excombatientes está asociada con un espectro más amplio de posiciones políticas, como pueden ser la defensa de la no-violencia o del pacifismo, la defensa del derecho a autodefensa, o la promoción de políticas de seguridad nacional anti-insurgencia, entre otros.

Fue así como, a lo largo del sondeo y diseño del estudio, pudimos articular nuevas preguntas sobre el desafío de posicionarse frente a los relatos del estudio y sobre las posibilidades de narrar y escuchar en el contexto socio-histórico del Chile actual: ¿Cuál es el imaginario nacional sobre la lucha armada y la lucha no-violenta en Chile, tanto en la actualidad como en el pasado? ¿Cómo informan estos imaginarios la capacidad de relatar y escuchar experiencias diversas de lucha social en Chile? ¿Cómo construimos y cómo recibimos relatos de acción no-violenta en pre-dictadura? ¿Cómo han influido en esto las políticas de verdad y reconciliación chilenas? ¿Qué espacios y roles ha preestablecido el proceso de reconciliación para quienes narran sus experiencias de lucha en el Chile del siglo XX? ¿Qué espacios y qué roles predeterminados existen para quienes relatan y escuchan experiencias chilenas de acción política actual?

Si bien responder a estas preguntas seriamente sería materia de otras investigaciones, enunciarlas nos sensibilizó sobre los tabúes, silencios, y narrativas preestablecidas relevantes a nuestro estudio (Andrews, 2007; 2014; Daiute, 2010; 2013; Duncan, Stevens y Sonn, 2012).

\subsection{Desafíos éticos en la escucha}

Una tercera veta en el proceso de sondeo y diseño del estudio fue la reflexión sobre desafíos éticos de trabajar con nuestros participantes en su calidad de adultos mayores que se consideran a sí mismos como figuras controversiales y que son además sobrevivientes de violencia política. Nos interesaba especialmente mitigar los 
potenciales riesgos asociados a las dinámicas de la academia hiper-productiva, contexto en el existe la tentación de desatender a la calidad de los procesos investigativos a favor de la rapidez y el cumplimiento de indicadores (Fardella-Cisternas, Sisto-Campos y Jiménez Vargas, 2017).

Como apoyo a la reflexión, aplicamos el marco ético desarrollado por Anderson y Muñoz-Proto (2016) a nuestro estudio, distinguiendo entre requerimientos éticos institucionales y responsabilidades éticas no normadas pero centrales a la investigación cualitativa de la justicia social. Dicho marco se centra en las complejidades de la confidencialidad como forma de protección de la privacidad (Folkman, 2010) y en el modo en que las investigaciones representan los participantes ante la audiencia de la investigación (Cahill, 2007).

Así, identificamos una serie de preguntas abiertas para promover el reconocimiento y el abordaje de las responsabilidades éticas no normadas que conllevaba el proyecto: ¿Bajo qué condiciones es ético estudiar los relatos de nuestros participantes? ¿Cuáles serían los riesgos y los beneficios de mantener la confidencialidad de los relatos, especialmente si los participantes buscasen difundirlos públicamente? ¿Qué resguardos deberíamos tomar para la protección de la privacidad de terceros mencionados en los relatos? ¿Cómo acompañar éticamente a los participantes en la tarea de decidir qué y cómo compartir con el equipo de investigación, con el público académico, y con el público en general? ¿Qué responsabilidades tenemos en la difusión de estos relatos más allá de lo académico? ¿Quiénes, bajo qué circunstancias y en qué formatos deben/pueden tener acceso a los relatos? ¿Quién decide esto? ¿Somos responsables los investigadores de representar de modo positivo a los participantes? ¿Qué responsabilidad tenemos de brindar a los participantes apoyo para que puedan publicar sus relatos si quisieran hacerlo?

Estas preguntas nos permitieron identificar dos desafíos éticos. Primero, debíamos responder al imperativo de realizar una escucha atenta a las complejidades, contradicciones, y tabúes que actualmente se negocian al narrar experiencias de lucha en Chile, buscando abordar y representar de manera humana y multidimensional las experiencias estudiadas. Segundo, y dada la condición controversial del tema y los participantes, debíamos explicitar nuestros intereses y puntos de vista en la interpretación, sin escondernos tras una mirada neutral, tras procedimientos rígidos, o tras una voz omnipresente.

\section{UN MARCO PARA LA ESCUCHA}

Como discutimos en el apartado anterior, el proceso de sondeo y diseño del estudio reveló desafíos técnicos y éticos relacionados al problema, población, contexto socio-histórico y académico del mismo. A raíz de estas conclusiones recurrimos a los aportes feministas del enfoque narrativo (Riessman, 2001), buscando un marco teórico-metodológico que permitiera abordar los desafíos que habíamos identificados.

Fue así que nos encontramos con el Método de la Guía de Escucha (MGE), desarrollado por Gilligan en la década de 1980 (Gilligan, Spencer, Weinberg y Bertsch, 2003; Kiegelmann, 2000; Sorsoli y Tolman, 2008) y aún sin un suficiente desarrollo en contextos hispanos. El MGE responde a las limitaciones de 
la codificación de contenidos de los estudios de Kohlberg sobre el desarrollo moral en mujeres, integrando influencias conceptuales de la música, la literatura, el feminismo y la psicología clínica (Gilligan, Spencer, Weinberg y Bertsch, 2003; Kiegelmann, 2000; Sorsoli y Tolman, 2008) con el fin de permitir una escucha detenida, recursiva y reflexiva (Doucet y Mauthner, 2008; Koelsh, 2015; Sorsoli y Tolman, 2008). Nuestro estudio está fuertemente inspirado en dos elementos centrales del MGE: una concepción dinámica y relacional de la subjetividad narrada; y el abordaje sistemático de estas características a través de la escucha activa, recursiva y reflexiva orientada por preguntas.

\subsection{Una concepción dinámica y relacional de la subjetividad narrada}

El MGE refleja una noción multivocal del yo (self), visibilizando contradicciones, tensiones y elementos contrapuntuales en un sujeto (Sorsoli y Tolman, 2008). La identidad social no responde a una unidad monolítica estable, racional y consistente, sino como un conjunto de elementos encarnados que emergen en relación al contexto y a otros (Doucet y Mauthner, 2008; Gilligan et al., 2003). Este yo se expresa de múltiples formas, siendo la voz una de ellas en tanto relación entre lo psíquico y lo corporal en la expresión humana (Sorsoli y Tolman, 2008). Se teoriza que en ciertos momentos puede hablar una sola voz del participante, y en otros momentos manifestarse otras voces en contraposición o complementariedad (Sorsoli y Tolman, 2008).

En comparación con el análisis de contenidos, el MGE facilita la escucha de silencios, tensiones, tabúes, contradicciones y otras complejidades que permean los relatos de experiencia e identidad. Este marco conceptual no fuerza el análisis hacia una coherencia de contenidos y temáticas en un relato, sino que da espacio para comprender las estructuras internas, los cambios, las inconsistencias y las tensiones que co-existen en las identidades, iluminando así la dinámica relación entre lo social y lo individual (Gilligan et. al, 2003; Kiegelmann, 2009).

Estas características hacen al MGE especialmente generativo en estudios sobre las tensiones, conflictos y complejidades que permean una subjetividad narrada (Gilligan et. al, 2003), sobre todo en torno a experiencias tabú (Koelsh, 2015), a conflictos (Kiegelmann, 2000), a estigmas sociales y a experiencias difíciles de contar (Sorsoli y Tolman, 2008). Así, el MGE ha informado numerosos estudios sobre juventud, deserción escolar, y desarrollo social adolescente en contextos de injusticas (Byrne, Canavan y Millar, 2009; Edwards y Weller, 2012; Koelsh, 2015). Respecto a nuestra temática de investigación, no existe gran aplicación del MGE, más allá un estudio sobre desvinculación de grupos armados (Villanueva O’Driscoll y Loots, 2014). 


\subsection{Escucha abierta, recursiva y reflexiva}

Además de ofrecer un marco teórico propicio, el MGE nos orientó hacia un abordaje sistemático de las ya mencionadas complejidades, tensiones y transformaciones presentes en los relatos del estudio. Autores como Kiegelmann (2000), explican que quien investiga debe ser capaz de identificar los distintos elementos que constituyen esa subjetividad narrada en el relato a través de un análisis abierto a lo emergente y complejo.

Se hace importante por esto la distinción entre un procedimiento predeterminado de análisis y una guía de análisis. Como lo indica su nombre, el MGE orienta a la persona que investiga en la implementación creativa, pertinente y flexible de ciertos momentos de análisis (Gilligan et. al, 2003). Estos momentos involucran la lectura reiterada del relato, cada vez focalizándose en un elemento o dimensión de la subjetividad narrada, como puede ser una voz particular (Doucet y Mauthner, 2008; Sorsoli y Tolman, 2008). Es así como el MGE promueve una escucha recursiva.

Algunas versiones del MGE privilegian la identificación de voces o dimensiones emergentes (Gilligan et al., 2003; Kiegelmann, 2000) mientras otras versiones trabajan desde voces predefinidas por quien investiga (Doucet y Mauthner, 2008). Gilligan propone, por ejemplo, una aproximación abierta a la escucha de la voz del sujeto narrado y la voz contra-puntual que emerge en tensión a la primera cuando se narran eventos significativos (Sorsoli y Tolman, 2008). Haciendo uso de este método se pudo, por ejemplo, identificar una relación dinámica entre dos voces que componían los relatos de mujeres estadounidenses sobre los dilemas morales, especialmente en relación a sexualidad: La voz del cuidado y la voz de la justicia (Gilligan et. al, 2003).

Doucet y Mauthner, por otra parte, proponen cuatro momentos de escucha con el fin de resaltar la relación entre subjetividad y sociedad (2008: 9). Un primer momento atiende a los elementos relacionales y reflexivos que constituyen las narrativas del estudio, combinando preguntas de la teoría fundamentada (¿Qué está pasando aquí?) con preguntas clásicas del análisis narrativo (¿Cuál es la trama, temáticas, personajes claves, etc.?; Doucet y Mauthner, 2008, p.8). Esto es similar al análisis narrativo que se focaliza en la descripción de la trama, de los eventos y de los personajes (Bernasconi, 2011; Daiute, 2013; Gibbs, 2012). En un segundo momento las autoras se basan en el concepto de narrativas ontológicas (Somers, 1994, en Doucet y Mauthner, 2008) para realizar un seguimiento de la subjetividad narrada a lo largo del texto, seleccionando las oraciones del relato en primera persona y analizando cuándo la persona habla desde el yo, el nosotros/as y el uno/a para resaltar cómo la persona se construye a lo largo el relato. En un tercer momento Doucet y Mauthner se focalizan en la voz experta, que comenta y opina sobre el relato desde la certeza. En cuarto lugar las autoras atienden a los aspectos relacionales del sujeto narrado, buscando iluminar cómo el sujeto se constituye como un sujeto-en-relación con otros y su mundo (2008, p. 9). 
Finalmente, el MGE insiste en una aproximación dialógica a través del concepto de escucha activa sobre el de lectura pasiva o análisis de transcripciones (Sorsoli y Tolman, 2008). Escuchar un relato es aproximarse a éste en un acto interpretativo y relacional en que dialogamos con la narrativa (Kiegelmann, 2000), utilizando la propia subjetividad en el proceso de investigación (Gilligan et. al, 2003; Walby, 2013). Para esto, la persona que trabaja con un relato va consignando sus propias reacciones, asociaciones y preguntas durante los varios momentos de escucha (Gilligan et. al, 2003).

\section{UN DISEÑO NARRATIVO CENTRADO EN LA ESCUCHA}

El diseño final del estudio combina técnicas reconocidas dentro del enfoque biográfico (Bernasconi, 2011; Cornejo, Mendoza y Rojas, 2008; Wertz, Charmaz, McMullen, Josselson, Anderson, y McSpadden, 2011;) con los elementos de la Guía de Escucha discutidos en el apartado anterior. El resultado es un diseño narrativo en el que la producción y trabajo con los relatos toma la forma de un proceso de escucha y aprendizaje. En este proceso conviven la escucha en profundidad de cada relato con una atención constante a los patrones de similitud y contraste entre los relatos que conforman la muestra, lo que Cornejo, Mendoza y Rojas (2008) llamarían un trabajo en simultáneo desde una lógica singular y una lógica transversal.

A continuación, describiremos el proceso completo de trabajo con cada participante, el cual contempla varios momentos de proceso. Cada momento se organiza en torno a una pregunta particular que orienta la escucha durante la preparación, conducción, y posterior análisis de una entrevista.

\subsection{Entrevista de sintonía y las condiciones para la escucha}

En esta primera entrevista del proceso corroboramos el cumplimiento de los criterios de inclusión y dialogamos sobre el proceso de consentimiento. La pregunta que orienta la escucha en este momento de proceso es: ¿Cuáles son las condiciones de mutua sintonía necesarias para la colaboración con esta persona y la escucha de su relato? El objetivo es conocerse en persona, establecer un mínimo de confianza, y prepararse para las sesiones posteriores. Buscamos también comunicar una actitud comprensiva hacia los desafíos que implicará el proceso con el siguiente guion:

...Si usted quiere puede prepararse para compartir su historia ... sabemos que hay mucho que contar y puede parecer un desafío, pero se hace más fácil si uno se concentra en las vivencias y eventos que han sido más significativos para uno/a. Si quiere puede traer anotaciones de apoyo. 


\subsection{Entrevista narrativa y escucha abierta}

En este segundo encuentro buscamos escuchar sin forzar, interrumpir o cuestionar; abiertos a la estructura, contenidos, silencios e incongruencias del relato que surja. La escucha en este momento del proceso está orientada por la siguiente pregunta: ¿Qué ocurre en este relato de acción política?

El encuadre busca neutralizar cualquier ánimo de debate o discusión en los participantes e investigadores:

Te hemos invitado a que nos cuentes, a grandes rasgos, el arco de tu trayectoria de acción política. No hay una forma correcta de contar el propio relato y esperamos que sientas libertad de contar como quieras y lo que quieras. A lo más podemos decir que un relato de vida típicamente cuenta la propia historia, pasando por los momentos más significativos para la persona. Nosotros escucharemos con sincero interés, sin interrumpir. [Nombre entrevistador/a 2] tomará notas de nombres o siglas que luego puedan ser difíciles de transcribir. Sin duda hay mucho que contar en una sola hora, así que te avisaremos cuando quede media hora de tiempo. No te preocupes si no entras en detalles hoy ya que en la siguiente entrevista vamos a profundizar. Tal vez quieras unos minutos para ordenar tus ideas ahora o durante el relato. Podemos empezar cuando quieras.

Si hubiera dificultades durante el relato, recalcamos nuestra disposición a la escucha (Ej. ¿Nos podrías contar que pasó en ese momento/después? ¿Cómo viviste ese momento?), evitando preguntas que lleven a la opinión, la justificación o el debate (Ej. ¿Qué opinas de eso que pasó? ¿Por qué crees que hiciste eso/ tomaste esa decisión?). Cerramos la entrevista insistiendo en las condiciones de la escucha para el resto del proceso:

Gracias por compartir tu historia y apoyar nuestro proyecto. Hemos aprendido sobre tu experiencia y lo seguiremos haciendo en las siguientes entrevistas. Te entregaremos el audio y la transcripción en la próxima entrevista. Hasta entonces estaremos atentos a cualquier clarificación o enmienda. Te invitamos a reunirnos en un mes para profundizar en el relato luego de escucharlo de modo más detenido.

\subsection{Escucha preliminar de giros y entrevista de profundización}

En la entrevista de profundización focalizamos la escucha en la pregunta central del estudio: ¿Cómo narra esta persona su tránsito entre diversos modos de lucha social? Antes de ejecutar la entrevista, la dupla entrevistadora y una tercera persona del equipo identifican los giros e inflexiones presentes en el relato de lucha social, respondiendo a las siguientes preguntas: ¿Cuál es el título de este giro? ¿Cuál es el antes y el después? ¿Cómo lo significa la persona? Luego 
de resolver diferencias de número y descripción de los giros de estas tres lecturas, construimos una pauta semi-estruturada con el siguiente encuadre:

Nos gustaría profundizar en tu relato y pedirte que nos ayudes a comprender mejor algunos momentos de transformación en tu trayectoria. Contamos con unos 45 minutos para hacerte algunas preguntas. Como la vez anterior, nuestro interés es escuchar tu relato, aunque esta vez tenemos algunas preguntas más específicas. Las vamos a ir mencionando en orden cronológico de acuerdo a la trayectoria que nos contaste. La idea es que nos puedas ir corrigiendo si comprendimos mal ¿Te surge alguna duda?

Como se ejemplifica en la Tabla 1, para cada momento de profundización identificamos una cita que compartimos con la persona con el fin de comunicar una escucha activa de lo ya compartido. Preparamos también preguntas que inviten a la profundización del relato en vez de la opinión o la justificación de lo hecho. Consignamos también temáticas que hemos identificado como centrales en la escucha preliminar de los giros para guiar nuestra escucha durante la entrevista.

Tabla 1. Ejemplo de Pauta Entrevista de Profundización

\begin{tabular}{|c|c|c|}
\hline $\begin{array}{l}\text { Giro a } \\
\text { profundizar }\end{array}$ & Consigna general/ cita & Temas de profundización \\
\hline $\begin{array}{l}\text { Inicio político } \\
\text { en año } 70\end{array}$ & $\begin{array}{l}\text { En la entrevista anterior } \\
\text { nos contaste que a partir } \\
\text { del año } 1970 \text { "viene } \\
\text { todo el periodo de la pre } \\
\text { adolescencia, del liceo, y } \\
\text { uno empieza a motivarse } \\
\text { por el tema político." Nos } \\
\text { gustaría escuchar más sobre } \\
\text { ese momento de tu juventud } \\
\text { en el que te motivaste por } \\
\text { lo político por primera } \\
\text { vez y te acercaste a las } \\
\text { juventudes de izquierda. } \\
\text { ¿Nos podrías contar más } \\
\text { sobre ese momento y cómo } \\
\text { lo viviste? }\end{array}$ & $\begin{array}{l}\text { Proyecto político inicial y primeras } \\
\text { experiencias con el trabajo político } \\
\text { no-armado: Nos gustaría escuchar } \\
\text { más sobre cómo comprendías tú ese } \\
\text { primer proyecto político a esa edad. } \\
\text { Diferenciación con otras opciones } \\
\text { de lucha: Nos contaste que las } \\
\text { opciones de lucha estaban, no había } \\
\text { que inventarlas. Nos gustaría oír } \\
\text { más sobre las opciones que se } \\
\text { presentaban en tu mundo para los } \\
\text { jóvenes de tu edad. ¿Cómo veías tú, } \\
\text { con ojos jóvenes, las opciones de } \\
\text { identidad política y participación que } \\
\text { se te ofrecían? }\end{array}$ \\
\hline
\end{tabular}

(Manuel, 61 años, Ex-liderazgo Movimiento de Izquierda Revolucionaria, actual liderazgo en movimientos socio-ambientales). 
Cabe mencionar que durante el diseño del estudio nuestro foco estaba en la escucha de los capítulos del relato, concebidos como un periodo de tiempo que está compuesto de eventos espacial y temporalmente contiguos y dotados de cierta unidad de significado (Mcadams, 1995). A poco andar comprendimos que para responder a nuestra pregunta de investigación sería necesario poner el énfasis en el paso entre un capítulo y otro, $\mathrm{o}$, mejor dicho, en los giros entre un tipo o momento de lucha social y otro.

\subsection{Entrevista de diálogo para corroborar la escucha}

Esta entrevista final toma la forma de diálogo en el que buscamos corroborar las interpretaciones preliminares sobre el relato de la persona. Nos basamos en la técnica de la Entrevista Activa Semiestructurada Individual (Hostein y Gubrium, 1995; Denzin, 2001), tomando un rol activo al presentar nuestras preguntas de investigación y proponer interpretaciones preliminares al participante. Nos orientan dos preguntas guía: ¿Cómo interpreta esta persona su propio relato? ¿Cómo respondería a nuestras preguntas de investigación? Iniciamos el diálogo con el siguiente encuadre:

Como te contamos inicialmente hoy buscamos dialogar contigo sobre las grandes preguntas de nuestro proyecto y pedirte que nos orientes en nuestra escucha de tu relato. A lo largo del proceso te hemos escuchado con interés, pero desde la ignorancia: somos de otra generación, hemos tenido vidas diferentes, nos parecemos en algunas cosas pero somos muy diferentes en otras. Por esto buscamos aprender de tu propia interpretación del relato y su significado en el Chile de hoy. Para inspirar el diálogo te iremos presentando las cuatro grandes preguntas que nos estamos haciendo sobre tu relato y pidiendo tu punto de vista sobre las interpretaciones preliminares a las hemos llegado. El espíritu del diálogo es que nos corrijas, profundices, opines, es decir, que te tomes la palabra a partir de lo que te vamos compartiendo.

Cabe mencionar sobre esta entrevista que el análisis narrativo puede ser lento y laborioso. El cronograma del estudio implicó llevar un volumen considerable de casos simultáneamente, limitando nuestra capacidad de elaborar interpretaciones finales entre la tercera y cuarta entrevista, ya que hacerlo habría implicado el transcurrir de muchos meses entre éstas. El trabajo con adultos mayores, sin embargo, nos llevó a valorar la cercanía temporal entre una y otra entrevista, optando por presentar interpretaciones preliminares. Inesperadamente, esto propició un diálogo más significativo en las entrevistas finales ya que tanto participantes y entrevistadores recordábamos en detalle lo ocurrido y dicho en entrevistas anteriores. $\mathrm{Al}$ mismo tiempo, considerar nuestras interpretaciones como preliminares permitió una mayor apertura a la escucha de interpretaciones distintas a las nuestras. 


\subsection{Selección de segmentos y escucha focalizada}

El proceso hasta ahora descrito generó relatos extensos sobre un rango de seis a ocho décadas de vida y acción política. Si bien el MGE promueve el trabajo con el relato en su totalidad, el volumen de información nos llevó a focalizar la escucha en los segmentos más relevantes a nuestro estudio, utilizando como apoyo nuestra adaptación del modelo de guía de escucha de Mauthner y Doucet (2008).

Comenzamos por seleccionar los segmentos del relato en donde se da cuenta de un giro o cambios en el abordaje de la lucha social: ¿Dónde en el relato da cuenta la persona de los cambios de rumbo en su trayectoria de lucha social y acción política? Para esto utilizamos como insumo el análisis realizado en preparación a la entrevista de profundización. El proceso genera una versión resumida del relato sobre la cual es posible realizar una escucha focalizada y recursiva. Para validar la escucha y resolver diferencias de criterio interpretativo se presentaron y trabajaron los casos ante el equipo en jornadas de validación.

En relación a la selección de los segmentos, utilizamos el criterio de 'lo mínimo posible:' Un segmento lo más breve posible que en sí mismo de cuenta tanto de la situación inicial, la transformación o inflexión, y la situación o rumbo resultante. La lectura en voz alta del segmento es una técnica simple y útil que permite corroborar en equipo si la selección da cuenta en sí misma del giro en cuestión, sin necesidad de referirse a la entrevista original. La selección referente a un giro puede estar compuesta por segmentos de la entrevista narrativa y/o de profundización.

Luego de la selección de segmentos, comenzamos con la escucha de la trama y temas presentes, generando memorándums que incluyen una breve descripción de los temas que le dan significado y un título a cada segmento. Por título entendemos una oración que dé cuenta del antes y el después en el desarrollo político de la persona. Al ser leídos en orden cronológico, el conjunto de títulos de los varios giros de un relato conforman una síntesis de la trayectoria de la persona.

Al trabajar con el relato de Sara (75 años, Movimiento de Izquierda Revolucionaria MIR, actual activista socio-ambiental) notamos la importancia de distinguir entre trama y tema con el fin de realizar efectivamente un análisis narrativo y no uno de contenido. Al ser Sara periodista, el tema de los medios de comunicación como herramienta política es transversal a todo su relato y particularmente relevante en el segundo y el tercer giro de su trayectoria. Dada esta coincidencia, fue especialmente importante que el título describiera la trama (¿Qué cambio ocurre aquí en la trayectoria de acción política?) reservando el análisis temático para el breve memorándum descriptivo (¿A qué temáticas recurre la persona para significar este giro?). En el caso de Sara, dimos al segundo giro el título de "cambia la acción política en la Iglesia Católica por el periodismo político' mientras que al tercero se le tituló 'deja el periodismo televisivo por un rol de liderazgo en las comunicaciones del MIR.' Esta forma de trabajo nos permitió consignar el significado narrativo único de giros que comparten una 
misma temática central. Por contraste, si estos y otros giros del relato compartieran el mismo título de 'las comunicaciones y la política' estaríamos realizando un análisis de contenido más que un análisis narrativo.

Habiendo titulado y descrito cada giro, volvimos sobre los segmentos seleccionados, preguntándonos por el sujeto político narrado en cada cual: ¿Sobre quién/quiénes trata este segmento del relato? ¿Cómo cambia este sujeto durante el segmento? Cuando estas respuestas no eran fáciles, recurrimos a la técnica de Gilligan en la que se aíslan las oraciones en primera persona singular y plural en un Poema del Yo (identidad individual) y Poema del Nos (identidad colectiva) para aislar visualmente los adjetivos, acciones, emociones y atributos a través de los que se construye una identidad en relación a otros y en el tiempo.

Finalmente, nos focalizamos en los comentarios del narrador sobre sobre las acciones políticas descritas en cada segmento, respondiéndonos: ¿Cómo evalúa esta persona, desde la mirada experta, los cambios narrados? ¿Qué controversias o debates públicos del relato sitúan a estas evaluaciones en el contexto Chileno?

La selección de segmentos y la escucha focalizada y reiterada de cada giro nos permitió acercarnos a los complejos significados de las trayectorias de acción política descritas en los relatos y su relación con la historia reciente de Chile. Significados que se plasman en un Resumen Interpretativo de cada relato, y que luego reconsideramos a la luz de las interpretaciones que los propios participantes ofrecieron en la entrevista de co-análisis. En dicho momento se enfatiza la atención a los patrones de similitud y diferencia a través de la técnica de comparación constante derivada de la teoría fundamentada y su aplicación al análisis de material narrativo (Charmaz y McMullen, 2011). Esto permite generar una respuesta global a la pregunta de investigación y avanzar también hacia una teorización comprensiva del fenómeno.

\section{BUENAS CONDICIONES PARA LA EJECUCIÓN DEL DISEÑO}

Es importante consignar que cada uno de estos momentos de proceso requiere de atención a las mejores condiciones para su ejecución. Durante el proceso muestreo, observamos que fue de gran importancia que el contacto inicial con potenciales participantes fuese a través de personas de su confianza con el fin de dar conocer el proyecto de un modo poco invasivo. Durante el proceso de entrevistas se nos evidenció la importancia de practicar la escucha reflexiva, consignando los posicionamientos, dudas, prejuicios y reacciones afectivas que emergían al escuchar los relatos (Gilligan et al., 2003). Esta práctica nos permitió visibilizar los desafíos éticos, técnicos y metodológicos del estudio, relacionados a las condiciones socio-históricas que influencian y limitan nuestra forma de escuchar.

Respecto de la colaboración con los participantes, identificamos seis puntos de especial importancia: (1) atender al posible requerimiento de las/los participantes de borrar o excluir del análisis o publicación ciertos pasajes de sus relatos; (2) usar pseudónimos para participantes y terceros mencionados quienes 
no fuesen figuras públicas; (3) realizar cierres respetuosos del proceso de participación y de la escucha de los relatos; (4) entregar los audios y transcripciones de las entrevistas a los participantes del estudio en un tiempo breve; (5) ofrecer la derivación a profesionales fuera del marco de la investigación de participantes que expresasen la necesidad de acompañamiento y escucha por parte de un psicólogo u otro profesional, lo cual no fue necesario; y (6) respetar la eventual decisión de los/las participantes de hacer públicos sus relatos fuera del marco de la investigación y atender a quienes quisieran dialogar con nosotros sobre las consideraciones a tener en cuenta en ese caso (Ej. Elaboración de una versión del relato para distribución pública, decisiones sobre confidencialidad, etc.).

También pudimos identificar numerosas condiciones técnicas que resguardan la calidad del proceso de producción de relatos. Las sesiones deben ser cortas, de 90 minutos como máximo, y en lugares silenciosos y libres de interrupciones familiares y laborales. Es importante que una misma dupla de investigadores acompañe el proceso completo con cada participante para que cada entrevista sea un espacio de escucha no sólo íntimo sino también dinámico. Es conveniente, además, que todas las sesiones ocurran dentro de tres meses desde la primera para que el diálogo se mantenga fresco y el vínculo activo. Para asegurar la calidad de las transcripciones, los relatos deben ser grabados en audio con al menos dos equipos, y transcritos dentro de dos semanas. Es útil que después de cada entrevista la dupla de investigación reflexione sobre sus aciertos, desafíos y descubrimientos, con la ayuda de una pauta de observaciones. Finalmente, es crucial dedicar suficiente tiempo para que la dupla, e idealmente una tercera persona del equipo, preparen la pauta para la siguiente entrevista, corroborando y validando sus interpretaciones preliminares y generando las condiciones para hacer de la siguiente sesión lo más provechosa posible.

\section{CONCLUSIONES}

El propósito del presente artículo ha sido contribuir al desarrollo de investigaciones narrativas y al diálogo sobre los desafíos y las decisiones metodológicas relativas al trabajo con relatos de lucha social. Tanto el estudio mismo como los objetivos del artículo se fundamentan en el rol de los relatos biográficos y otras narrativas de experiencia en tanto formas de construir y comunicar el conocimiento humano (Bruner, 1990). En este marco, hemos presentado el proceso de diseño de un estudio centrado en las historias de vida de activistas de la tercera edad quienes participaron de la izquierda armada chilena en su juventud y cuentan con largas trayectorias de activismo a lo largo de sus vidas.

El artículo refleja importantes avances en el estudio narrativo de las dimensiones psicosociales de la paz y los conflictos, los que han iluminado los relatos e vida en tanto aspecto central de la experiencia humana en su dimensión sociohistórica y psicosocial (Andrews, 2007; Atkinson, 2001; Bernasconi, 2011; Bolívar y Domingo, 2006; Capella, 2013; Chase, 2015; Cornejo, 2006; Cornejo, Mendoza y Rojas, 2008; Daiute, 2010; 2013; Daiute y Lightfoot, 2004; De la 
Rey, 2011; Doucet y Mauthner, 2008; Duncan, Stevens y Sonn, 2012; Josselson y Lieblich, 1993; Law y Bretherton, 2015); 2014).

Crear espacios para la narración de experiencias investigativas nos parece importante, para dejar atrás una cierta una cierta reverencia disciplinar hacia el pensamiento lógico-formal (Bruner, 1990) como única forma válida de integrar y comunicar experiencias investigativas. En vista que la gran mayoría de los requerimientos institucionales y los códigos de ética están pensados desde una lógica cuantitativa, hemos buscado aportar al desarrollo las dimensiones éticometodológicas de la investigación cualitativa sobre fenómenos relacionados a la justicia social y los relatos de vida.

El objetivo del diseño que hemos presentado es múltiple. Primero, hemos buscado dar cabida al carácter dinámico y complejo de las subjetividades narradas de los participantes del estudio a través de una escucha abierta que atienda a cómo negocian silencios, tabúes, estigmas y relatos predefinidos en torno a la acción política, la violencia y la acción no-violenta, abordando de modo sistemático su complejidad. Segundo, hemos intentado dar oportunidades para que el proceso de relato y escucha se desarrolle, madure y profundice en el tiempo, generando instancias para que el carácter relacional de los relatos se manifieste. Finalmente, el diseño ha buscado busca visibilizar cómo la práctica de la narración y de la escucha nos posicionan en el contexto socio-histórico chileno presentándonos importantes desafíos éticos y técnicos.

A través de una adaptación original del modelo de guía de escucha de Mauthner y Doucet (2008), hemos buscado producir descripciones empíricas y herramientas teórico-conceptuales sobre los tipos de narrativas que dan significado a las experiencias de acción política de los participantes, con énfasis en las transformaciones de sus identidades y formas de acción en el tiempo. Estos resultados darán pie a conclusiones sobre los complejos significados que se construyen sobre la violencia y la no-violencia desde la acción política de la izquierda post-dictatorial y las implicancias de estos significados para los repertorios de acción política en situaciones contemporáneas de conflicto socio-ambiental, educacional y comunicacional. En su potencial aplicación estos resultados pueden informar la formación de líderes constructores de paz, fortaleciendo así la capacidad ciudadana de participación activa, propositiva y constructiva en situaciones de conflictos y malestar social.

\section{BIBLIOGRAFIA}

Anderson, S. \& Muñoz-Proto, C. (2016) Ethical Requirements and Responsibilities in Video Methodologies: Considering Confidentiality and Representation in Social Justice Research. Social Personality Psychology Compass, 10(7), pp. 377-389.

Andrews, M. (2007): Shaping History: Narratives of political change, Cambridge, Cambridge University Press.

Andrews, M. (2014): Narrative Imagination and Everyday Life, Oxford, Oxford University Press. 
Atkinson, P. (2001): “The Life Story Interview", en Handbook of interview research, London, Sage Publications, pp. 121-140.

Bellei, C., Cabalin, C. y Orellana, V. (2014): The 2011 Chilean student movement against neoliberal educational policies, Studies in Higher Education, 39, 4, pp. 426-440.

Bernasconi, O. (2011): Aproximación narrativa al estudio de fenómenos sociales: principales líneas de desarrollo, Acta Sociológica, 56, pp. 9-36.

Bolívar, A. y Domingo, J. (2006): La investigación biográfica y narrativa en Iberoamérica, Forum: Qualitative Social Research, 7, 4.

Byrne, A., Canavan, J. y Millar, M. (2009): Participatory research and the voice-centred relational method of data analysis: is it worth it?, International Journal of Social Research Methodology, 12, 1, pp. 67-77.

Bruner, J. (1990): Culture and Human Development: A New Look, Human Development, 33, 6, pp. 344-355.

Cahill, C. (2007): Repositioning ethical commitments: Participatory action research as a relational praxis of social change, ACME: An International E-Journal for Critical Geographies, 6, 3, pp. 360-373.

Capella, C. (2013): "Una propuesta para el estudio de la identidad con aportes del análisis narrativo", Psicoperspectivas, 12, 2, pp. 117-128.

Wertz, F., Charmaz, C., McMullen, L. M., Josselson, R., Anderson, R. y McSpadden, E. (2011): Five ways of doing qualitative analysis, New York, The Guilford Press.

Chase, S. (2015): "Investigación narrativa", en Métodos de recolección y análisis de datos, Barcelona, Gedisa, pp. 58-112.

Cornejo, M. (2006): El enfoque biográfico: Trayectorias, desarrollos teóricos y perspectivas, Psykhe, 15, 1, pp. 95-106.

Cornejo, M., Mendoza, F. y Rojas, R. (2008): La investigación con relatos de vida: Pistas y opciones del diseño metodológico, Psykhe, 17, 1, pp. 29-39.

Daiute, C. (2010): Human development and political violence, Cambridge, Cambridge University Press.

Daiute, C. (2013): Narrative Inquiry: A Dynamic Approach, Thousand Oaks, Sage Publications.

Daiute, C. y Lightfoot, C. (2004): Narrative analysis: studying the development of individuals in society, Thousand Oaks, Sage Publications.

De la Rey, C. (2011): "Narrative Analysis", en The Encyclopedia of Peace Psychology, Malden, Wiley Blackwell Publishing, pp. 685-687.

Denzin, N. (2001). The Reflexive interview and performative social science, Qualitative Research, 1, 1, pp. 23-46.

Donoso Jiménez, J. (2013): Violencia política en Chile entre el pueblo Mapuche y el gobierno del presidente Ricardo Lagos, Revista de Relaciones Internacionales, Estrategia y Seguridad, 8, 2, 63-93.

Doucet, A. y Mauthner, N. (2008): What can be know and how? Narrated subjects and the listening guide, Qualitative Research, 8, 3, pp. 399-409.

Duncan, N., Stevens, G. y Sonn, C. (2012): Of narratives and nostalgia. Peace and conflict, Journal of Peace Psychology, 18, pp. 205-2013.

Edwards, R., y Weller, S. (2012): Shifting analytic ontology: Using I-Poems in qualitative longitudinal research, Qualitative Research, 12, 2, pp. 202-217.

Fardella-Cisternas, C., Sisto Campos, V., \& Jiménez Vargas, F. (2017). La transformación de la universidad y los dispositivos de cuantificación. Estudos de Psicologia 34(3), pp. 435-448. 
Fine, M. (2012): Resuscitating critical psychology for "revolting" times, Journal of Social Issues, 68, 2, pp. 416-438.

Flick, U. (2014): La gestión de la calidad en investigación cualitativa, Madrid, Morata.

Folkman, S. (2010): "Privacy and confidentiality", en Ethics in Research with Human Participants, Washington, American Psychological Association, pp. 48-54.

Hatibovic Díaz, F., Sandoval Moya, J. y Cárdenas Castro, M. (2012): Posiciones de sujeto y acción política universitaria: Análisis de discurso de estudiantes de universidades de la región de Valparaíso, Última Década, 20, 37, pp. 111-134.Garcés, M. (2011): Chilean Social Movements in Confrontation with neoliberalism, en Neoliberalism's Fractured Showcase: Another Chile is Possible, Leiden, Brill, pp. 219-248.

Garcés, M. (2012): El despertar de la sociedad: Los movimientos sociales en América Latina y Chile, Santiago, LOM ediciones.

Gibbs, G. (2012): El análisis de datos cualitativos en investigación cualitativa, Madrid, Ediciones Morata.

Gilligan, C., Spencer, R., Weinberg, K. y Bertsch, T. (2003): “On the listening guide: A voice centered relational method", en Qualitative research in psychology, Washington, American Psychological Association, pp. 157-172.

Goicovic, I. (2004): La implacable persistencia de la memoria. Reflexiones en torno al Informe de la comisión de prisión política y tortura, RHA, 2, 2, pp. 73-91.

Goicovic, I. (2012): "El Movimiento de Izquierda Revolucionaria (MIR) y la irrupción de la lucha armada en Chile, 1965-1990", En Historia oral e historia política, izquierda y lucha armada en América Latina, 1960-1990, Santiago, LOM ediciones, pp. 159-189.

Guerrero Antequera, M. (2006): El conjuro de los movimientos sociales en Chile neoliberal, Revista Venezolana de economía y ciencias sociales, 12, 2, pp. 147-156.

Holstein, J. y Gubrium, J. (1995): The active interview, Thousand Oaks, Sage Publications.

Jaimovich, D., Gonzalez, B., Calbucura, J., del Valle, N. y Maldonado, C. (2018): Conflictos sociales y ambientales en Chile, Iberoamericana, 28, 67, pp. 205-230.

Josselson, R. y Lieblich, A. (1993): The narrative study of lives, Thousand Oaks, Sage Publications.

Koelsh, L. (2015): I Poems: Evoking Self, Qualitative Psychology, 2, 1, pp. 96-107.

Kiegelmann, M. (2000): Qualitative Psychological research using the voice-approach, Forum: Qualitative Social Research, 1, 2, pp. 1-8.

Kiegelmann, M. (2009): Making Oneself Vulnerable to Discovery. Carol Gilligan in Conversation With Mechthild Kiegelmann, Forum: Qualitative Social Research, 10, 2.

Kool, V. (1993): Nonviolence: social and psychological issues, Lanham, University Press of America.

Law, S. y Bretherton, D. (2015): “Qualitative research methods in peace psychology”, en Methodologies in peace psychology, New York, Springer, pp. 89-114.

Lincoln, Y. (2012): "Los comités de conducta ética y el conservadurismo metodológico", en El campo de la investigación cualitativa, Barcelona, Gedisa, pp. 332-361.

Lira, E. (2010): Trauma, Duelo, Reparación y Memoria, Revista de Estudios Sociales, 36 , pp. 14-28.

Lira, E. (2013): Algunas reflexiones a propósito de los 40 años del golpe militar en Chile y las condiciones de la reconciliación política, Psykhe, 22, 2, pp. 5-18. 
Lozoya, I. (2012): “Chile: violencia política y transición a la democracia”, en Historia oral e historia política, izquierda y lucha armada en América Latina, 1960-1990, Santiago, LOM ediciones, pp. 191-212.

Mcadams, D. (1995): The life story interview, Evanston, North Western University.

Martínez, L., Peñaloza, P. y Valenzuela, C. (2012): Civic commitment in young activists: Emergent processes in the development of personal and collective identity, Journal of Adolescence, 35, 3, pp. 474-484.

Moulian, T. (2002): Chile actual. Anatomía de un mito, Santiago, LOM ediciones.

Muñoz Proto, C. (2014). "What We Are, Where We Are Headed": A Peace March Visits an Ex-torture Center. In Symbols that Bind, Symbols that Divide: The Semiotics of Peace and Conflict. (pp. 39-59). R. Phillips DeZalia \& S. Moeschberger (Eds.). New York: Springer. (Peace Psychology Book Series edited by Dan Christie, ISSN: 21975779.)

Muñoz Proto, C., Devoto Lyon, A., Villar Castillo, C. \& Battistella, M. (2013). Memoscopio: Producing usable and collectively owned knowledge about the World March for Peace and Nonviolence. Journal of Social Issues 64(9), pp. 754-770.

Muñoz Proto, C. \& Opotow, S. (2012). Justice, activity, and narrative: Studying the World March. In Psychological Components of Sustainable Peace. (pp. 177-196). P. Coleman \& M. Deutsch (Eds.). New York: Springer. (Peace Psychology Book Series edited by Dan Christie, ISSN: 2197-5779.)

Pérez, C. (2012): "De la guerra contra Somoza a la guerra contra Pinochet: La experiencia internacionalista revolucionaria en Nicaragua y la construcción de la fuerza militar propia del Partido Comunista de Chile", En Historia oral e historia política: izquierda y lucha armada en América Latina, 1960-1990, Santiago, LOM Ediciones, pp. $213-244$.

Reardon, B. y Cabezudo, A. (2002): Learning to abolisch war: Teaching toward a culture of peace, New York, Hague Appeal for Peace.

Reyes, M. J., Muñoz, J., y Vázquez, F. (2013): Políticas de memoria desde los discursos cotidianos: La despolitización del pasado reciente en el Chile actual, Psykhe, 22, 2, pp. 161-173.

Riessman, C. (2001): “Analysis of personal narratives", en Handbook of interview research, London, Sage Publications, pp. 695-710.

Salazar, G. (2013): Villa Grimaldi (Cuartel Terranova). Historia, Testimonio, Reflexión, Santiago, LOM ediciones.

Salazar, G. (2015): La enervante levedad histórica de la Clase Política Civil (Chile, 1900-1973), Santiago, Debate.

Sixsmith, J., Boneham, M. y Goldring, J. (2003): Accessing the Community: Gaining Insider Perspectives From the Outside, Qualitative Health Research, 13, 4, pp. 578589.

Sorsoli, L. y Tolman, D. (2008): "Hearing voices: Listening for multiplicity and movement in interview data", en Handbook of emergent methods, New York, The Guilford Press, pp. 495-517.

Villanueva O'Driscoll, J. y Loots, G. (2014): Tracing subjetive drives: A narrative approach to study youth's engagement with and disengagement from armed groups in Colombia, Qualitative Research in Psychology, 11, 4, pp. 365-383.

Walby, K. (2013): Institutional ethnography and data analysis: making sense of data dialogues, International Journal of Social Research Methodology, 16, 2, pp. 141-154. 
